\title{
Effet de l'incorporation des protéines du lactosérum dénaturées et microparticulées dans le cheddar jeune
}

\author{
Yolaine Lebeuf*, Christophe Lacroix, Paul Paquin
}

Centre de recherche en sciences et technologie du lait (STELA), Faculté des sciences de l'agriculture et de l'alimentation, université Laval, Sainte-Foy, Québec, Canada, G1K 7P4

(Reçu le 27 septembre 1996 ; accepté le 4 septembre 1997)

\begin{abstract}
Effect of incorporation of denatured and microparticulated whey protein in young Cheddar cheese. Two whey protein concentrates (WPC) heat-denatured at $\mathrm{pH} 4.2$ (WPC 4.2) and at pH 6.7 (WPC 6.7) and microparticulated were used for the production of Cheddar cheese. Three micro-productions of Cheddar were made in triplicate: one with a control milk and the other two with milks in which the WPC 4.2 and WPC 6.7 ingredients were respectively added. The total amount of proteins was increased by $0.5 \mathrm{~kg} / 100 \mathrm{~kg}$ of milk $(0.5 \%)$. The experimental cheeses had a significant higher yield, i.e., $4.3 \%$ with WPC 4.2 and $5.2 \%$ with WPC 6.7 , than the control cheese, at a $37 \%$ constant moisture. Cheeses manufactured under these procedures were lower in fat and had a higher moisture content than the control cheese. Electronic microscopy shows that the control cheese had a more compact structure than the experimental cheeses. (c) Inra/Elsevier, Paris.
\end{abstract}

denaturation / whey protein / microfluidization / cheese yield

Résumé - Deux concentrés de protéines du lactosérum (CPL) dénaturés par traitement thermique à pH 4,2 (CPL 4,2) et à pH 6,7 (CPL 6,7) et microparticulés ont été utilisés pour la fabrication de fromage cheddar. Trois micro-productions de cheddar ont été effectuées en triplicata avec un lait témoin, un lait additionné de l'ingrédient CPL 4,2 et un lait additionné de l'ingrédient CPL 6,7 pour augmenter la quantité totale de protéines de $0,5 \mathrm{~kg} / 100 \mathrm{~kg}$ de lait $(0,5 \%)$. Les fromages expérimentaux ont présenté un rendement significativement plus élevé que le fromage témoin, avec une augmentation moyenne de 4,3\% (CPL 4,2) et 5,2\% (CPL 6,7) sur une base d'humidité constante de $37 \%$. Une teneur en lipides plus basse et un contenu en humidité plus élevé ont été obtenus pour les fromages constitués de laits supplémentés. L'examen en microscopie électronique révèle une structure légèrement plus compacte pour le fromage témoin comparé aux fromages expérimentaux. (C) Inra/Elsevier, Paris.

dénaturation / protéine du lactosérum / microfluidisation / rendement fromager

* Correspondance et tirés à part. 


\section{INTRODUCTION}

L'industrie fromagère montre un intérêt grandissant pour l'utilisation et le développement de nouvelles applications des concentrés de protéines du lactosérum (CPL), en particulier en vue d'incorporer les protéines du lactosérum dans le fromage [18]. La raison principale est économique. Le lactosérum contient, environ, $20 \%$ des protéines du lait. L'incorporation des protéines du lactosérum dans le fromage augmente le rendement fromager et ainsi la rentabilité de la fabrication fromagère. Cependant, la qualité des fromages peut être affectée car les propriétés des protéines du lactosérum diffèrent de celles des caséines [21]. Elles ont la capacité de retenir plus d'eau, ce qui affecte la texture et la saveur du fromage [22]. L'incorporation des protéines du lactosérum présente également un intérêt nutritionnel car les protéines sont riches en lysine, tryptophane et acides aminés soufrés [11].

Les protéines du lactosérum peuvent être incorporées sous forme native ou dénaturée. Trois méthodes de base ont été développées pour l'incorporation des protéines de lactosérum dans le fromage [21]. La concentration du lait par ultrafiltration (UF) permet de standardiser les protéines du lait. Le traitement thermique du lait pour dénaturer les protéines du lactosérum provoque une complexation de ces protéines avec les caséines qui s'accompagne d'une rétention dans le caillé. Enfin, la dénaturation des protéines du lactosérum avant de les additionner au lait de fromagerie permet d'emprisonner les protéines devenues insolubles dans la matrice fromagère.

La fabrication de fromage à partir de lait concentré par UF est maintenant bien établie commercialement pour un nombre limité de fromages [20]. Les avantages de I'UF pour augmenter les rendements fromagers sont moins importants pour les fromages à faible taux d'humidité comme le cheddar que ceux à taux plus élevé [20]. Dans le cas du fromage cheddar, environ $30 \%$ des protéines solubles d'un lait concentré 5-1 devraient théoriquement être retenues dans le fromage représentant une augmentation potentielle du rendement de 5 à $8 \%$ au maximum [21]. En revanche, cela va occasionner des problèmes au niveau de la coagulation du lait, de la texture du produit et des caractéristiques d'affinage. Les fromages fermes et semi-fermes vont avoir une texture beaucoup plus molle. Le traitement thermique du lait pour favoriser la dénaturation des protéines solubles et leur incorporation dans le fromage est fortement limité dans le cas des pâtes pressées, comme le cheddar, par les problèmes de coagulation, d'égouttage et par les défauts occasionnés au produit. Le chauffage du lait provoque des interactions entre la $\kappa$-caséine et la $\beta$-lactoglobuline par des ponts disulfures ce qui affecte la coagulation par la présure. La dernière méthode semble être la plus prometteuse car le procédé de fabrication fromagère n'est pas perturbé. La dénaturation et l'isolation des protéines du lactosérum avant de les additionner au lait de fromagerie peut éviter les problèmes causés par la complexation des caséines avec les protéines du lactosérum au cours des traitements [23]. Certaines études ont déjà été réalisées sur l'addition de CPL dénaturés liquides et en poudre au lait pour la production de fromage cheddar $[1,4$, $5,8]$. En général, le rendement fromager était augmenté. En revanche, les fromages présentaient un taux élevé d'humidité, la perte en matières grasses lors de la transformation était augmentée et de mauvaises saveurs se développaient lors de l'affinage.

Pour être incorporées dans le fromage, les protéines du lactosérum dénaturées et dispersées dans le lait, doivent être emprisonnées dans le gel de caséines formé par f'action de la présure [23]. La dénaturation thermique de CPL produit des agré- 
gats protéiques. La taille de ces agrégats peut avoir un effet néfaste lors de la production fromagère s'ils sont de trop grosse taille. Ils affectent alors la coagulation du lait et l'égouttage du caillé. Selon Lelièvre [23], les agrégats protéiques devraient avoir une taille entre 0,1 et $10 \mu \mathrm{m}$ pour ne pas perturber la matrice de polymère. Les particules ayant une taille plus petite et sphérique devraient se comporter, jusqu'à un certain point, comme des globules de gras dans la matrice fromagère [25]. Lelièvre [23], démontre l'intérêt d'additionner au lait un CPL dénaturé et microparticulé pour la production d'un fromage Mozzarella. Une augmentation de rendement d' environ $20 \%$ a été obtenue. Le fromage avait des propriétés sensorielles, de fusion et d'étirement comparables à celles du témoin, malgré une augmentation de l'humidité et une diminution du rapport lipides/extrait sec.

Dans cette étude, nous proposons d'utiliser deux ingrédients en poudre à base de protéines du lactosérum concentrées, dénaturées thermiquement dans des conditions acide et neutre et microparticulées à l'aide d'un microfluidisateur. L'effet de l'incorporation de ces ingrédients protéiques a été examiné sur le rendement fromager, la composition et la texture du fromage cheddar jeune ainsi que sur la microstructure de la matrice fromagère.

\section{MATÉRIEL ET MÉTHODES}

\subsection{Matériel}

Le lactosérum doux utilisé pour la fabrication des ingrédients protéiques provenait de la fromagerie Saint-Michel Archange (Québec, Canada). Il a été recueilli après le premier soutirage partiel lors de la production du cheddar. Il a été écrémé sur une écrémeuse Westfalia Separator Model MP1254 (Oelde, Allemagne) et ensuite pasteurisé à $75^{\circ} \mathrm{C}$ pendant $17 \mathrm{~s} \mathrm{à}$ l'aide d'un pasteurisateur à plaque APV modèle MC1622 type HX (Paraflow, Angleterre).

Le lait cru utilisé pour la fabrication des fromages cheddar était un lait de grand mélange, pasteurisé à $63^{\circ} \mathrm{C}$ pendant $30 \mathrm{~min}$. Le ferment lactique de type DVS (direct vat starter, lot \# 900), la solution commerciale de chlorure de calcium $\left(\mathrm{CaCl}_{2}\right)$ et la solution commerciale de présure $50-50$ composée d'extrait de chymosine et de pepsine ont été obtenus de Chr. Hansen's Laboratory (Milwaukee, WI, États-Unis).

\subsection{Préparation des ingrédients protéiques}

Un lactosérum écrémé à une température de $45^{\circ} \mathrm{C}$ a été concentré par ultrafiltration $(5 \mathrm{x})$ sur une membrane spiralée ayant un seuil de coupure de $5000 \mathrm{Da}$ (Système Abcor, Kotch Membrane Systems Inc., Wilimgton, ÉtatsUnis), puis diafiltré à deux reprises. Le CPL obtenu contient $84,4 \%$ de protéines sur base sèche. Les protéines du CPL ont été dénaturées thermiquement dans des conditions acide et neutre. Le CPL avait un pH de 6,7 (CPL $6,7)$. Avant traitement, une partie du produit à pH 6,7 a subi un ajustement de $\mathrm{pH}$ à 4,2 (CPL 4,2 ) avec une solution aqueuse d'acide citrique $50 \%(\mathrm{p} / \mathrm{v})$ de qualité alimentaire. Un traitement thermique à $95^{\circ} \mathrm{C}$ pendant 3 min a été appliqué aux deux CPL dans un autocuiseur (Groen MFG CO. Elk Grove, IL, États-Unis). Après traitement, le $\mathrm{pH}$ a été réajusté à 6,7 pour le CPL dénaturé à $\mathrm{pH} 4,2$ avec de l'hydroxyde de sodium $50 \%$ (p/v). L'homogénéisation a été faite par microfluidisation à l'aide d'un microfluidisateur model $\mathrm{M}-110^{\mathrm{TM}}$ (Microfluidic Corporation, Boston, MA, ÉtatsUnis) à un débit maximum de $36 \mathrm{~L} / \mathrm{h}$, une pression de $76 \mathrm{MPa}$ et une température de $25^{\circ} \mathrm{C}$. Une étape de concentration par microfiltration, avec une membrane Ceraflo (Millipore, Bedford, MA, États-Unis) ayant un seuil de coupure de $0,45 \mu \mathrm{m}$, a été effectuée afin d'augmenter le taux de matières solides totales à $20 \%$ pour faciliter le séchage par atomisation avec un Mini Spray Dryer Buchi 190 (Brinkmann, Westbury, NY, Etats-Unis) [25].

\subsection{Fabrication fromagère}

À partir de lait de grand mélange, trois productions différentes ont été effectuées avec un lait témoin, un lait additionné de l'ingrédient CPL 4,2 et un lait additionné de l'ingrédient 
CPL 6,7. Le taux d'incorporation des ingrédients protéiques a été calculé pour augmenter la quantité totale des protéines du lait de $0,5 \mathrm{~kg} / 100 \mathrm{~kg}$ de lait $(0,5 \%)$. Les ingrédients protéiques en poudre ont été incorporés au lait cru avant la pasteurisation, ce qui permet une meilleure répartition et hydratation des ingrédients. Trois répétitions ont été réalisées à partir d'une micro-production fromagère. Pour chaque répétition, trois lots de fromages ont été fabriqués dans une expérience en bloc complet. Deux $\mathrm{kg} \pm 1 \mathrm{~g}$ de lait pasteurisé et refroidi à $32{ }^{\circ} \mathrm{C}$ ont été pesés dans des plats de plastique (Freshware ${ }^{\mathrm{MC}}$, capacité de $2,7 \mathrm{~L}$ ) et placés dans un bain thermostaté. Les laits à une température de $32^{\circ} \mathrm{C}$ ont été inoculés avec le ferment lactique (DVS) à une concentration de $0,16 \%(\mathrm{v} / \mathrm{v})$. Après $50 \mathrm{~min}$, le $\mathrm{CaCl}_{2}$ et la présure ont été ajoutés aux laits à une concentration chacune de $0,025 \%(\mathrm{v} / \mathrm{v})$. Après 30 min, le coagulum a été coupé en cubes avec un couteau muni de fils d'acier inoxydable espacés de $7 \mathrm{~mm}$. Après une période de repos de $10 \mathrm{~min}$ le caillé a été chauffé graduellement jusqu'à $38^{\circ} \mathrm{C}$ sur une période de $40 \mathrm{~min}$ avec agitation. Un soutirage partiel d'environ $50 \%$ du lactosérum a été effectué et le caillé a été maintenu à $38^{\circ} \mathrm{C}$ pendant 30 min avant le soutirage final. Le caillé a subi une cheddarisation jusqu'à ce que le $\mathrm{pH}$ atteigne 5,3 avec une inversion périodique des blocs. Les blocs ont alors été coupés à l'aide d'un couteau bien aiguisé en morceaux d'environ $30 \times 15 \times 20$ $\mathrm{mm}$. Cinq grammes de sel ont été ajoutés à chaque production. Le caillé a été placé dans des moules Kadova (brevet canadien 845035 ) et une pression de $300 \mathrm{~Pa}$ a été appliquée pendant $16 \mathrm{~h}$ à la température de la pièce.

\subsection{Analyse chimique}

Les analyses chimiques des fromages ont été faites immédiatement après pressage. Une pointe totalisant un quart de meule a été découpée, râpée et bien mélangée pour uniformiser l'échantillonnage. Le pourcentage de lipides des fromages a été déterminé par la méthode Babcok [24]. L'azote total des fromages, des laits, des lactosérums et des poudres de CPL a été mesuré par la méthode Kjeldahl [16]. Le contenu en lipides des laits, des lactosérums et des poudres de CPL a été déterminé par la méthode de Mojonnier [3]. L'azote caséique et l'azote non protéique (NPN) ont été déterminés en duplicata par la méthode Kjeldahl
[16]. Les caséines ont été sélectivement précipitées à leur $\mathrm{pH}$ isoélectrique de 4,6 , éliminées par filtration et le contenu en azote noncaséique du surnageant a été dosé par Kjeldahl. La fraction NPN a été obtenue par précipitation des protéines et des protéoses-peptones à l'acide trichloroacétique à une concentration finale de $12 \%$. Le facteur de convertion de 6,38 a été utilisé pour convertir l'azote en protéines. Le taux de dénaturation des protéines des ingrédients protéiques a été évalué par mesure de l'indice de solubilité de l'azote [17]. Le contenu en matières solides totales des laits, des lactosérums et des fromages a été déterminé en quadruplicata selon la méthode de l'AOAC [3]. Le $\mathrm{pH}$ des fromages a été obtenu avec une électrode à solide (Radiometer America Inc., Westlake, $\mathrm{OH}$, États-Unis).

\subsection{Analyse de la taille des particules}

L'évaluation du diamètre moyen et de l'étendue de la distribution des agrégats protéiques a été effectuée sur les ingrédients liquides avant séchage par la méthode de spectroscopie d'auto-corrélation photonique (PCS) utilisant un Nicomb Submicron Particle Sizer System (Model 370, Pacific Scientific, Manlo Park, CA, États-Unis) [26].

\subsection{Analyse microscopique}

Des observations au microscope optique de marque Zeiss \#64448 (Iena, Allemagne) ont été effectuées sur les poudres de protéines du lactosérum dénaturées et microparticulées réhydratées à 2,5\% (p/v). La taille moyenne des particules des poudres réhydratées a été mesurée à partir des photos.

Les fromages ont été examinés par microscopie électronique à balayage (SEM) Les fromages ont été coupés en forme de prismes d'approximativement $1 \times 1 \times 15 \mathrm{~mm}$. Quatre à six échantillons ont été fixés dans une solution aqueuse de glutaraldehyde $2,8 \%$ à $6{ }^{\circ} \mathrm{C}$ pendant 24 h et ensuite déshydratés dans une série de solutions d'éthanol $(20,40,60,80,96$, $100 \%$ d'éthanol). Ils ont été par la suite dégraissés dans du chloroforme et ensuite dans une solution pure d'éthanol. Les échantillons ont été rapidement congelés à $-150^{\circ} \mathrm{C}$ dans du Fréon 12 et transférés dans l'azote liquide ce qui a provoqué la fracture des échantillons. 
Les fragments ont été décongelés dans de l'éthanol absolu $\left(20^{\circ} \mathrm{C}\right)$ et ensuite séchés par du dioxide de carbone. Les fragments ont été montés sur un ruban adhésif double-face fixé aux pièces du SEM et ils ont été recouverts d'une couche d'or $(20 \mathrm{~nm})$. Ils ont été examinés à l'aide d'un microscope électronique à balayage de marque Zeiss DSM940 (Iena, Allemagne). Les photographies ont été prises avec un film Kodak T-Max 100 ASA.

\subsection{Calcul des rendements fromagers}

Le rendement actuel est défini comme le nombre de $\mathrm{kg}$ de fromages produits à partir de $100 \mathrm{~kg}$ de lait.

Le rendement ajusté est calculé sur une base de $37 \%$ d'humidité, en tenant compte des matières solides du lactosérum, à l'aide de la formule [15] :

$Y(\operatorname{adj}): Y($ act $) \times \frac{1-\text { WSCsef(act) }-M(\text { act })}{1-\text { WSCsef(std) }-M(\text { std })}$ avec :

$-Y$ : rendement fromager $(\mathrm{kg} / 100 \mathrm{~kg}$ de lait) ; -WSCsef : fraction de matières solides du sérum dégraissé dans le fromage, en tenant compte de l'effet d'exclusion des solutés dû aux protéines du fromage ;

$-M$ : fraction de l'humidité dans le fromage $(0,37)$;

- std : composition du fromage ajustée à une composition standard ;

- act : rendement et composition du fromage actuel sans ajustement.

Les taux de rétention de lipides et des protéines du lait dans le fromage ont été calculés selon les formules suivantes :
$\%$ rétention de lipides $=$ masse des lipides dans le fromage $\times 100$ masse des lipides dans le lait

$\%$ rétention protéines $=$ $\underline{\text { masse des protéines dans le fromage } \times 100}$ masse des protéines dans le lait

\subsection{Analyse statistique}

Une analyse de variance (Anova) a été réalisée pour tester la signification des différences des paramètres de composition physico-chimique et des rendements fromagers entre les trois types de laits utilisés. Un test de WallerDuncan a été utilisé pour vérifier si les différences $(p<0,05)$ sont significatives.

\section{RÉSULTATS ET DISCUSSION}

\subsection{Ingrédients protéiques microparticulés}

La composition des concentrés de protéines du lactosérum (CPL) dénaturés et microparticulés, sous forme de poudre, est présentée au tableau I. L'ingrédient CPL 4,2 est composé de $79,9 \%$ de protéines alors que le CPL 6,7 en contient $82,9 \%$. Les deux ingrédients ont été produits à partir du même CPL. L'ajout d'acide citrique et d'hydroxyde de sodium $(\mathrm{NaOH})$ pour l'ajustement du $\mathrm{pH}$ du CPL 4,2 a eu un effet diluant ce qui peut expliquer une quantité plus faible de protéines dans 1'ingrédient CPL 4,2. Le taux de dénaturation des protéines de l'ingrédient

Tableau I. Composition des ingrédients protéiques microparticulés en poudre.

Table I. Composition of microparticulated protein powder.

\begin{tabular}{cccc}
\hline Ingrédients & $\begin{array}{c}\text { Lipides } \\
(\%)\end{array}$ & $\begin{array}{c}\text { Protéines } \\
(\%)\end{array}$ & $\begin{array}{c}\text { Dénaturation } \\
\text { des protéines } \\
(\%)\end{array}$ \\
\hline CPL 4,2 & 8,2 & 79,9 & 88,5 \\
CPL 6,7 & 7,9 & 82,9 & 82,1 \\
\hline
\end{tabular}


CPL 4,2 est de $88,5 \%$ et de $82,1 \%$ pour le CPL 6,7. La quantité de protéines du lactosérum dénaturées est affectée par le $\mathrm{pH}$ pendant le chauffage [12]. La zone où les protéines sont les plus sensibles à la chaleur se situe entre les $\mathrm{pH} 4$ et 6 . La dénaturation est immédiatement suivie d'agrégation et de précipitation [7, 12]. La réduction de la charge nette des protéines près du point isoélectrique $(\mathrm{pH} 4,6)$ va minimiser les répulsions électrostatiques intermoléculaires ce qui va faciliter l'agrégation des protéines par des liens hydrophobes [28]. On observe une baisse de la dénaturation protéique pour des $\mathrm{pH}$ supérieurs à 6,5 car à ces $\mathrm{pH}$, la solubilité des protéines est élevée [12]. Il se produit également une stabilisation de la molécule par la création de ponts disulfures et par une réactivité accrue des groupements thiols aux $\mathrm{pH}$ supérieurs à 6,7 [12]. Les effets peuvent expliquer que le taux de dénaturation des protéines du CPL 4,2 soit plus élevé que celui du CPL 6,7.

La microfluidisation a réduit la taille des agrégats protéiques formés lors du traitement thermique pour dénaturer les protéines. Une analyse par PCS a permis de déterminer que les agrégats protéiques étaient devenus de fines particules ayant une taille se situant entre 1 et $10 \mu \mathrm{m}$. Des observations au microscope optique à un grossissement de $40 \mathrm{x}$ ont été effectuées sur les poudres réhydratées des ingrédients protéiques (figures $I a$ et $I b$ ). La taille moyenne des particules du CPL 6,7 est de $5,2 \pm 2,8 \mu \mathrm{m}$ en comparaison avec celle du CPL 4,2 qui est de 7,6 $\pm 3,0 \mu \mathrm{m}$. Selon Lelièvre [23], les agrégats protéiques devraient avoir une grosseur entre 0,1 et $10 \mu \mathrm{m}$ pour ne pas perturber le réseau caséique formé lors de la fabrication fromagère. De plus, il faut que l'ingrédient ait une forme sphérique pour obtenir un bon ingrédient protéique [27]. Les particules des deux CPL $(6,7$ et 4,2$)$ apparaissent de forme sphérique (figures $1 a$ et $1 b$ ). Les particules du CPL 4,2 réhy- draté ont une dimension légèrement plus grande que celles de l'ingrédient CPL 6,7. Langton et Hermansson [19] ont observé que la structure d'un gel de CPL formé à pH 4,5 était composé de particules sphériques de taille plus grande qu'un gel formé à $\mathrm{pH}>6,0$. La microstructure et les propriétés physiques des protéines du lactosérum sont affectées par le $\mathrm{pH}$ et les conditions du traitement thermique [19]. De plus, Buchheim et Jelen [9] ont aussi observé par microscopie électronique, qu'un CPL dénaturé à $\mathrm{pH} 4,5$ formait de larges agrégats compacts et bien définis alors qu'un CPL dénaturé à $\mathrm{pH} 6,5$ produisait des agrégats beaucoup plus petits et moins denses.

\subsection{Effets des ingrédients protéiques microparticulés additionnés au lait de fromagerie}

\subsubsection{Composition des laits de fromagerie}

La composition des laits utilisés pour la fabrication fromagère est présentée au tableau II. La quantité de protéines totales a été augmentée de $0,5 \%$ pour les laits additionnés des ingrédients protéiques par rapport au témoin non enrichi. La méthode d'analyse des caséines a également dosé les ingrédients protéiques microparticulés dans les laits enrichis ce qui explique les valeurs plus élevées en caséines relativement au lait témoin bien qu'aucun ajout de caséines n'ait été réalisé. Ainsi le lait témoin et les laits additionnés de CPL 6,7 et CPL 4,2 présentaient des concentrations en caséines de $2,57 \pm$ $0,08 \%, 3,02 \pm 0,08$ et $2,99 \pm 0,09 \%$, respectivement. Au cours de la précipitation des caséines par l'acide acétique $\left(\mathrm{CH}_{3} \mathrm{COOH}, 10 \% \mathrm{v} / \mathrm{v}\right)$, les protéines du lactosérum dénaturées microparticulées ont précipité en même temps que les caséines. Ceci démontre que les protéines du sérum microparticulées ne sont plus 

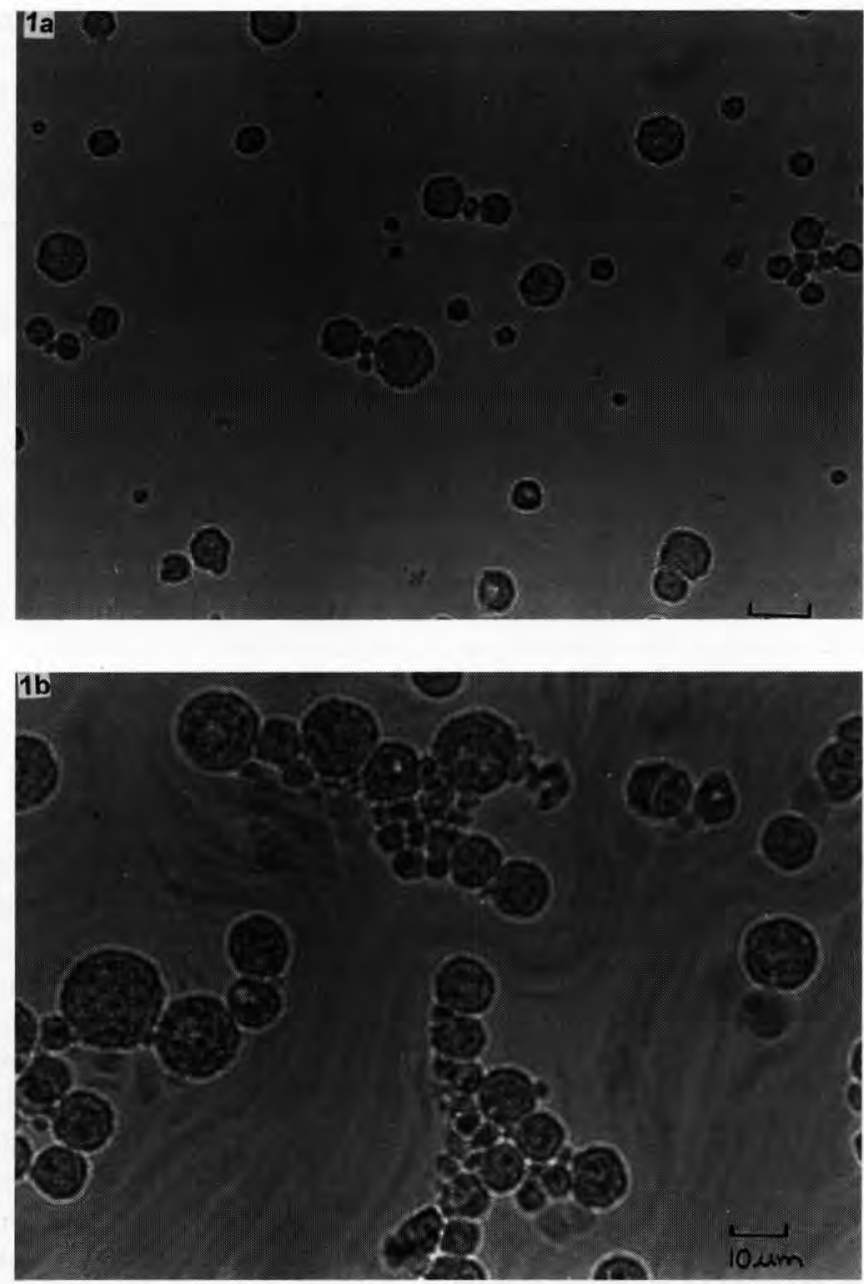

Figure 1. Examen en microscopie optique des ingrédients protéiques microparticulés réhydratés (40 X) (a) CPL 6,7 et (b) CPL 4,2.

Figure 1. Optical microscopy of microparticulated protein ingredients powder rehydrated $(40 \mathrm{X})$ (a) WPC 6.7 and (b) WPC 4.2.

solubles. Alors, elles devraient être retenues dans le réseau caséique lors de la fabrication fromagère. Un bilan de matière a été effectué pour déterminer par calcul la quantité de caséines dans les laits expérimentaux. Le lait additionné du CPL 6,7 contient $2,57 \pm 0,08 \%$ de caséines et celui du CPL 4,2, 2,53 $\pm 0,06 \%$ de caséines.
La différence entre ces résultats et les valeurs d'analyse des caséines correspond approximativement à la quantité d'ingrédients protéiques microparticulés ajoutée au lait, soit $0,45 \pm 0,03 \%$ pour le CPL 6,7 et $0,46 \pm 0,03 \%$ pour le CPL 4,2 . La quantité totale de matières solides totales des laits additionnés des ingrédients micro- 
Tableau II. Composition moyenne du lait témoin et des laits enrichis avec les ingrédients protéiques microparticulés $(n=3)$.

Table II. Composition of control milk and milks fortified with microparticulated protein ingredients $(n=3)$.

\begin{tabular}{|c|c|c|c|c|c|c|}
\hline \multirow[t]{2}{*}{ Laits } & \multicolumn{2}{|c|}{ Témoin } & \multicolumn{2}{|c|}{ CPL 6,7} & \multicolumn{2}{|c|}{ CPL 4,2} \\
\hline & $\mathrm{X}$ & SD & $\mathrm{X}$ & SD & $\mathrm{X}$ & SD \\
\hline Protéines totales (\%) & 3,19 & 0,05 & 3,70 & 0,08 & 3,68 & 0,04 \\
\hline Caséines (\%) & 2,57 & 0,08 & 3,02 & 0,08 & 2,99 & 0,09 \\
\hline $\mathrm{NPN}^{1}(\%)$ & 0,18 & 0,00 & 0,20 & 0,02 & 0,21 & 0,02 \\
\hline Lipides (\%) & 3,46 & 0,21 & 3,64 & 0,01 & 3,39 & 0,22 \\
\hline $\begin{array}{l}\text { Matières solides } \\
\text { totales }(\%)\end{array}$ & 12,15 & 0,07 & 12,65 & 0,07 & 12,68 & 0,16 \\
\hline
\end{tabular}

${ }^{1} \mathrm{NPN}$ : azote non protéique.

${ }^{1} \mathrm{NPN}$ : non protein nitrogen.

particulés a augmenté de $0,50 \%$ pour le CPL 6,7 et de $0,53 \%$ pour le CPL 4,2 par rapport au lait témoin. La composition des laits additionnés de l'ingrédient CPL 6,7 et de l'ingrédient CPL 4,2 est relativement semblable.

\subsubsection{Composition des fromages}

L'addition de protéines du lactosérum dénaturées et microparticulées au lait de fromagerie augmente de façon significative $(p<0,05)$ l'humidité des fromages (tableau III). L'humidité des fromages augmente en moyenne de $4,80 \%$ et $5,56 \%$ pour les laits additionnés des ingrédients CPL 6,7 et CPL 4,2 par rapport au fromage témoin. Cet effet de l'enrichissement en CPL du lait de fromagerie sur l'humidité des fromages peut être expliqué par le fait que les protéines du lactosérum dénaturées ont une plus grande capacité à lier l'eau que les caséines [4]. On n'observe pas de différence significative dans la quantité de protéines totales entre le fromage témoin et les fromages expérimentaux. En revanche, une diminution significative des lipides de $13 \%$ est observée pour les fromages expérimentaux par rapport au fromage témoin. La baisse des lipides des fromages expérimentaux serait due essentiellement à la composition (rapport protéines/lipides) des laits enrichis par rapport au lait témoin. L'enrichissement en protéines des laits expérimentaux provoque un débalencement du rapport protéines/lipides. Ce rapport protéines/lipides n'a pas été standardisé à un rapport constant ce qui peut expliquer cette perte en lipides des fromages additionnés des ingrédients protéiques microparticulés. L'ajout des CPL ne semble pas avoir modifié le procédé de fabrication des fromages. Les temps de fabrication et l'évolution du $\mathrm{pH}$ étaient comparables au fromage témoin. De plus, les caillés semblaient visuellement avoir la même fermeté au moment du coupage. En revanche, en modifiant le procédé de fabrication fromagère, soit le temps de coagulation du lait et le chauffage du caillé, cela permettrait possiblement de diminuer les 
Tableau III. Composition moyenne du fromage témoin et des fromages cheddar additionnés des ingrédients protéiques et rétention du gras et des protéines dans les fromages $(n=3)$.

Table III. Composition of control cheese and Cheddar cheese fortified with microparticulated protein ingredients and fat and protein retention in the cheese $(n=3)$.

\begin{tabular}{|c|c|c|c|c|c|c|}
\hline \multirow[t]{2}{*}{ Laits } & \multicolumn{2}{|c|}{ Témoin } & \multicolumn{2}{|c|}{ CPL 6,7} & \multicolumn{2}{|c|}{ CPL 4,2} \\
\hline & $\mathrm{X}$ & SD & $X$ & SD & $\mathrm{X}$ & SD \\
\hline Humidité (\%) & $34,78^{a}$ & 0,14 & $39,58^{\mathrm{b}}$ & 0,88 & $40,34^{b}$ & 0,48 \\
\hline Lipides (\%) & $34,91^{\mathrm{a}}$ & 0,25 & $30,16^{\mathrm{b}}$ & 0,29 & $30,08^{b}$ & 0,62 \\
\hline Protéines (\%) & $25,41^{\mathrm{a}}$ & 0,27 & $25,52^{\mathrm{a}}$ & 0,68 & $24,76^{\mathrm{a}}$ & 0,31 \\
\hline $\mathrm{L} / \mathrm{ES}^{1}(\%)$ & $53,5^{\mathrm{a}}$ & 0,50 & $49,9^{b}$ & 0,79 & $50,4^{\mathrm{b}}$ & 0,78 \\
\hline $\mathrm{P} / \mathrm{ES}^{2}(\%)$ & $38,9^{\mathrm{a}}$ & 0,33 & $42,2^{\mathrm{c}}$ & 0,53 & $41,4^{b}$ & 0,23 \\
\hline $\mathrm{pH}$ & $5,10^{\mathrm{a}}$ & 0,02 & $5,09^{\mathrm{a}}$ & 0,07 & $5,15^{\mathrm{a}}$ & 0,10 \\
\hline Rétention lipides (\%) & $95,8^{\mathrm{a}}$ & 1,19 & $89,3^{\mathrm{b}}$ & 0,69 & $89,3^{\mathrm{b}}$ & 0,69 \\
\hline Rétention protéines (\%) & $76,8^{\mathrm{a}}$ & 0,63 & $75,9^{\mathrm{a}}$ & 1,32 & $74,7^{b}$ & 0,69 \\
\hline
\end{tabular}

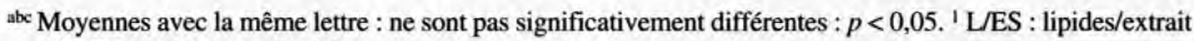
sec. ${ }^{2} \mathrm{P} / \mathrm{ES}$ : protéines/extrait sec.

abc Means with the same letter are not significantely different $P<0,05 .{ }^{1} \mathrm{LES}$ : fat/dry matter. ${ }^{2} \mathrm{P} / \mathrm{ES}$ : proteins/dry matter.

différences d'humidité entre le fromage témoin et les fromages expérimentaux et de diminuer les pertes dans le lactosérum. Le $\mathrm{pH}$ final des fromages expérimentaux est comparable au fromage témoin. L'acide citrique utilisé pour la production de l'ingrédient CPL 4,2 n'a pas d'effet sur la fabrication et la composition des fromages. Pendant la dénaturation du CPL, l'acide citrique a chelaté les minéraux dont le calcium présent dans le CPL. L'acide citrique participe à l'agrégation des protéines du lactosérum pendant le traitement thermique ce qui le rend non disponible dans le lait de fromagerie pour chelater le calcium. De plus, on n'observe pas de différence significative de composition entre les fromages faits avec l'ingrédient CPL 6,7 et l'ingrédient CPL 4,2.

\subsubsection{Composition des lactosérums de fromagerie}

L'analyse de la composition du lactosérum donne des informations indirectes sur la qualité de la production fromagère. On retrouve une quantité plus élevée de protéines et de matières solides totales pour les lactosérums expérimentaux comparativement à celui du témoin (tableau $I V)$. Une augmentation de la concentration moyenne de protéines de $0,23 \%$ est observée dans le lactosérum du fromage fait avec le CPL 6,7 et de $0,22 \%$ pour celui du CPL 4,2, possiblement des protéines solubles. Cette augmentation est nettement inférieure au niveau d'enrichissement en protéines de lactosérum dénaturées et microparticulés des laits de fromagerie, de $0,5 \%$. Ce résultat démontre 
Tableau IV. Composition moyenne des lactosérums de fromagerie $(n=3)$.

Table IV. Composition of cheese whey $(n=3)$.

\begin{tabular}{|c|c|c|c|c|c|c|}
\hline \multirow[t]{2}{*}{ Laits } & \multicolumn{2}{|c|}{ Témoin } & \multicolumn{2}{|c|}{ CPL 6,7 } & \multicolumn{2}{|c|}{ CPL 4,2} \\
\hline & $\mathrm{X}$ & SD & $\mathrm{X}$ & SD & $\mathrm{X}$ & SD \\
\hline Protéines totales (\%) & 0,81 & 0,07 & 1,04 & 0,01 & 1,03 & 0,02 \\
\hline $\mathrm{NPN}^{1}(\%)$ & 0,28 & 0,05 & 0,33 & 0,03 & 0,35 & 0,04 \\
\hline Lipides (\%) & 0,34 & 0,06 & 0,42 & 0,01 & 0,44 & 0,06 \\
\hline $\begin{array}{l}\text { Matières solides } \\
\text { totales }(\%)\end{array}$ & 6,71 & 0,06 & 6,99 & 0,07 & 7,10 & 0,07 \\
\hline
\end{tabular}

${ }^{1} \mathrm{NPN}$ : azote non protéique.

${ }^{1} \mathrm{NPN}$ : non protein nitrogen.

qu'une partie importante de ces protéines a été retenue dans la matrice fromagère. La quantité de lipides moyenne varie de $0,34 \% \pm 0,06$ pour le lactosérum témoin à $0,42 \% \pm 0,01$ et $0,44 \% \pm 0,06$ pour les lactosérums additionnés des ingrédients CPL 6,7 et CPL 4,2. Il y a perte de lipides à l'égouttage dû à une perte de la qualité du coagulum. L'ajout des protéines du lactosérum dénaturées et microparticulées augmente la quantité de particules dans la matrice aux dépens des caséines nécessaires à former la matrice fromagère ce qui provoque les pertes en protéines et lipides dans le lactosérum.

\subsubsection{Rendement fromager}

Le rendement fromager est influencé par la composition du lait en particulier sa teneur en lipides et en caséines, la composition finale du fromage, les pertes de lipides, de protéines et de fines dans le lactosérum et la technique de fabrication fromagère. Un facteur très important des rendements fromagers est l'humidité du fromage. Une augmentation de $1 \%$ de l'humidité d'un fromage contenant $37 \%$ d'humidité augmentera le rendement de $1,87 \%$ [13]. L'humidité des fromages expérimentaux est significativement plus élevée que celle du fromage témoin (tableau III), ce qui occasionne une augmentation des rendements fromagers. Le rendement actuel est significativement plus faible pour le fromage témoin comparativement aux fromages expérimentaux. Une augmentation moyenne de $14,48 \%$ et de $15,20 \%$ du rendement actuel a été observée pour les fromages fabriqués avec les laits additionnés des ingrédients protéiques CPL 6,7 et CPL 4,2 (tableau V). Le rendement ajusté a été utilisé pour corriger les variations du rendement causées par l'humidité des fromages [14]. Le rendement ajusté à une humidité constante du fromage ( $37 \%$ ) en tenant compte des matières solides du lactosérum a été calculé (tableau V). Selon Emmons [14], il est important d'inclure les matières solides du lactosérum lorsque la différence d'humidité est causée par des niveaux de l'égouttage différents du caillé et lorsque la différence d'humidité est grande. Les fromages expérimentaux présentent un rendement ajusté significativement plus élevé que le fromage témoin, avec une augmentation en moyenne de 5,23\% avec l'ingrédient CPL 6,7 et de 4,32\% avec l'ingrédient CPL 4,2 (tableau V). Cette augmentation 
Tableau V. Effet de l'addition des ingrédients protéiques sur les rendements fromagers $(n=3)$.

Tableau V. Effect of incorporation of whey proteins denaturated and microparticulated on the cheese yield $(n=3)$.

\begin{tabular}{|c|c|c|c|c|c|c|c|c|}
\hline & \multicolumn{4}{|c|}{ Rendement actuel } & \multicolumn{4}{|c|}{ Rendement ajusté $^{1}$} \\
\hline & \multirow{2}{*}{$\begin{array}{c}\text { Rendement } \\
(\mathrm{kg})\end{array}$} & \multicolumn{2}{|c|}{ Différence } & \multirow[t]{2}{*}{ SD } & \multirow{2}{*}{$\frac{\text { Rendement }}{(\mathrm{kg})}$} & \multicolumn{2}{|c|}{ Différence } & \multirow[t]{2}{*}{ SD } \\
\hline & & (kg) & $(\%)$ & & & (kg) & $(\%)$ & \\
\hline Témoin & $9,60^{\mathrm{a}}$ & - & - & 0,12 & $9,94^{\mathrm{a}}$ & - & - & 0,06 \\
\hline CPL 6,7 & $10,99^{b}$ & 1,39 & 14,48 & 0,08 & $10,46^{\mathrm{b}}$ & 0,52 & 5,23 & 0,10 \\
\hline CPL 4,2 & $11,06^{b}$ & 1,46 & 15,20 & 0,09 & $10,37^{b}$ & 0,43 & 4,32 & 0,10 \\
\hline
\end{tabular}

${ }^{\mathrm{ab}}$ Moyennes suivies de la même lettre : ne sont pas significativement différentes ; $p<0,05 .{ }^{1}$ Rendement ajusté à une composition standard de fromage de $37 \%$ d'humidité, en tenant compte des matières solides du lactosérum.

ab Means with the same letter are not significantely different; $P<0,05$. $^{\text {I }}$ Yield is adjusted at a standard moisture composition of cheese of $37 \%$ with fraction fat-free whey solids in cheese.

des rendements ajustés s'explique par la présence de protéines du sérum dénaturées et microparticulées dans les laits enrichis de CPL. Une partie des ingrédients protéiques microparticulés est retenue dans la matrice fromagère ce qui augmente les rendements ajustés des fromages expérimentaux. Les microparticules hydratées sont emprisonnées dans le caillé et se comporteraient comme les globules de gras dans la matrice de protéine [2]. Dans notre étude, la quantité ajoutée d'ingrédient protéique microparticulé était d'environ 6 $\mathrm{g} / \mathrm{kg}$ de lait. Banks et Muir [5] qui utilisaient une plus grande quantité de leur CPL dénaturés, soit $40 \mathrm{~g} / \mathrm{kg}$ de lait ont observé une augmentation du rendement ajusté pour l'humidité de $7 \%$. Les protéines du lactosérum utilisées de notre étude ont un taux de dénaturation élevé et une taille de particules réduite ce qui augmente leur rétention dans les fromages.

Il est généralement reconnu qu'environ $70-77 \%$ des protéines totales du lait peuvent être retenues dans le fromage [10]. Dans le tableau III, une diminution significative de la rétention des protéines est observée pour le fromage fait avec l'ingrédient CPL 4,2 par rapport au fromage témoin et à celui enrichi par le CPL 6,7 alors qu'on n'observe pas de différence significative entre ces deux derniers. La rétention des lipides est significativement plus faible pour les fromages expérimentaux que pour le fromage témoin (tableau III). Cette perte en lipides est attribuée au fait que les ingrédients protéiques microparticulés additionnés au lait de fromagerie sont emprisonnés dans le réseau caséique. Ils agissent jusqu'à un certain point comme la matière grasse. Une meilleure rétention de la matière grasse dépend du rapport caséines/lipides du lait de fromagerie qui est de 0,70 pour le cheddar [6]. Le rapport caséines/lipides + les ingrédients protéiques microparticulés des laits enrichis est de $0,63 \pm 0,02$ pour le lait additionné du CPL 6,7, de $0,66 \pm 0,04$ pour le lait additionné du CPL 4,2 et de $0,74 \pm 0,04$ pour le lait témoin. Donc, le ratio caséines/lipides + ingrédients protéiques microparticulés du lait de fromagerie est beaucoup plus faible pour les fromages expérimentaux. Ce faible rapport 
dans les laits enrichis occasionnerait la formation d'un coagulum fragile et des pertes importantes en lipides et protéines lors de l'égouttage du caillé. Les caséines ne peuvent pas emprisonner tout ces éléments dans le réseau caséique. Il est donc évident que dans le cas des traitements expérimentaux, la caséine est diluée dans le coagulum et le fromage par l'ajout des ingrédients protéiques microparticulés. Il en résulte des propriétés d'égouttage moins bonnes que pour le fromage témoin, un caillé hétérogène et friable et des pertes plus importantes en lipides et protéines dans le sérum. Toutefois, malgré les pertes de lipides et de protéines, les fromages expérimentaux ont un rendement fromager nettement supérieur au fromage témoin. Pour éliminer la perte en lipides et protéines des fromages expérimentaux, il faudrait diminuer la quantité de lipides du lait en fonction de la quantité d'ingrédients protéiques microparticulés ajouté. Cela permettrait de maintenir un rapport caséines/lipides + ingrédients protéiques microparticulés standard pour la fabrication fromagère.

\subsubsection{Microstructure des fromages}

La microscopie électronique à balayage (SEM) a permis d'illustrer les différences de microstructure entre le fromage témoin et les fromages expérimentaux. Les figures $2 a$ et $3 a$ représentent la matrice protéique d'un cheddar conventionnel. Une structure uniforme et compacte est observée. Les espaces vides dans la matrice protéique indiquent la localisation de l'eau et des lipides. Sur la figure $3 a$, on note la présence de bactéries lactiques contenues dans le réseau protéique. Les figures $2 b$ et $3 b$ représentent le fromage cheddar fabriqué avec le lait additionné de l'ingrédient CPL 6,7. Les protéines du lactosérum dénaturées et microparticulées ont été retenues dans la matrice fromagère. $\mathrm{La}$ structure globulaire des particules incorporées est bien préservée. La taille réduite des agrégats protéiques a permis de les intégrer dans le réseau caséique. Le réseau protéique est légèrement plus ouvert et moins compact pour le fromage fait avec l'ingrédient CPL 6,7 qu'avec le cheddar témoin. La présence des agrégats de protéines du lactosérum dénaturées et microparticulées modifie la structure du fromage cheddar. Les protéines du lactosérum dénaturées et microparticulées ayant la capacité de retenir l'eau rendent l'étape d'égouttage des fromages plus difficile ce qui explique un réseau de protéines plus lâche. Ce réseau de protéines moins compact expliquerait aussi la perte en lipides et protéines des fromages expérimentaux.

Les figures $2 c$ et $3 c$ représentent le fromage cheddar fabriqué avec l'ingrédient CPL 4,2. Les particules ont aussi conservé leur forme sphérique. La figure $3 c$ permet d'observer à un grossissement plus élevé des particules sphériques microfluidisées. Cette figure révèle que les particules sont composées d'une association de plusieurs petits agrégats globulaires de protéines du lactosérum dénaturées et microparticulées. Selon Buchheim et Jelen [9], la dénaturation par la chaleur provoque la formation d'agrégats submicroscopiques de tailles variables qui sont la structure des agrégats microscopiques. On observe aussi que le réseau protéique formé avec le CPL 4,2 est plus ouvert que celui du fromage témoin. En revanche, l'ingrédient CPL 4,2 semble mieux s'intégrer dans le réseau protéique que l'ingrédient CPL 6,7. La structure du fromage fait avec le CPL 4,2 est moins ouverte et plus uniforme que celle du fromage fait avec le CPL 6,7. On observe aussi de petits agrégats de protéines dénaturées et des particules de diamètre plus grand que $10 \mu \mathrm{m}$ dans le fromage CPL 4,2.

L'addition des protéines du lactosérum dénaturées et microparticulées modifie la structure des fromages. Le réseau de caséines est moins uniforme et les cavités où l'eau est retenue sont plus grandes 

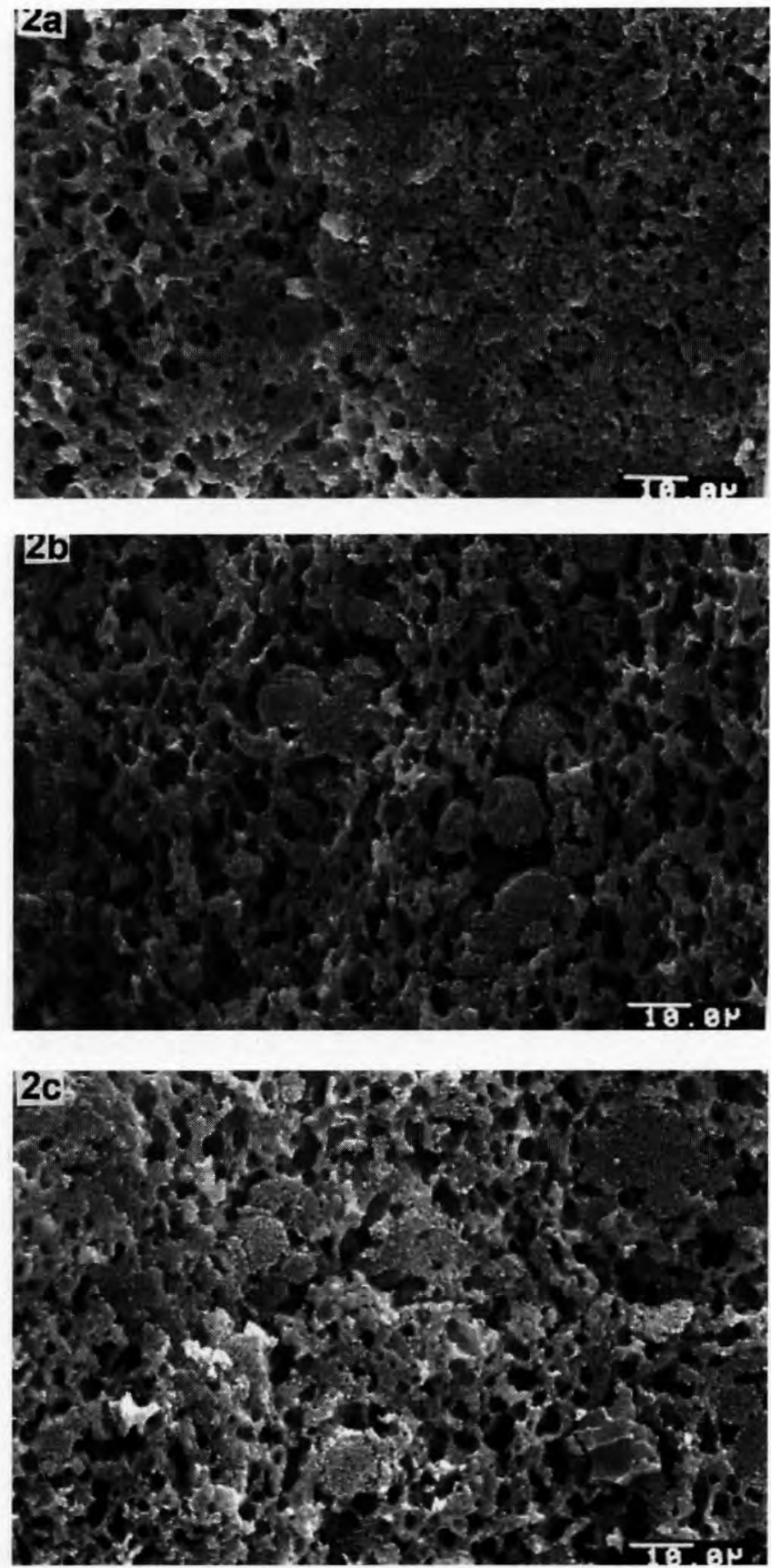

Figure 2. Microstructure des fromages additionnés des ingrédients protéiques microparticulés par SEM (1000 X) (a) fromage témoin, (b) fromage CPL 6,7 et (c) fromage CPL 4,2.

Figure 2. Microstructure of cheese fortified with whey proteins microparticulated by SEM $(1000 \mathrm{X})(\mathbf{a})$ cheese control (b) cheese WPC 6.7 and (c) cheese WPC 4.2. 

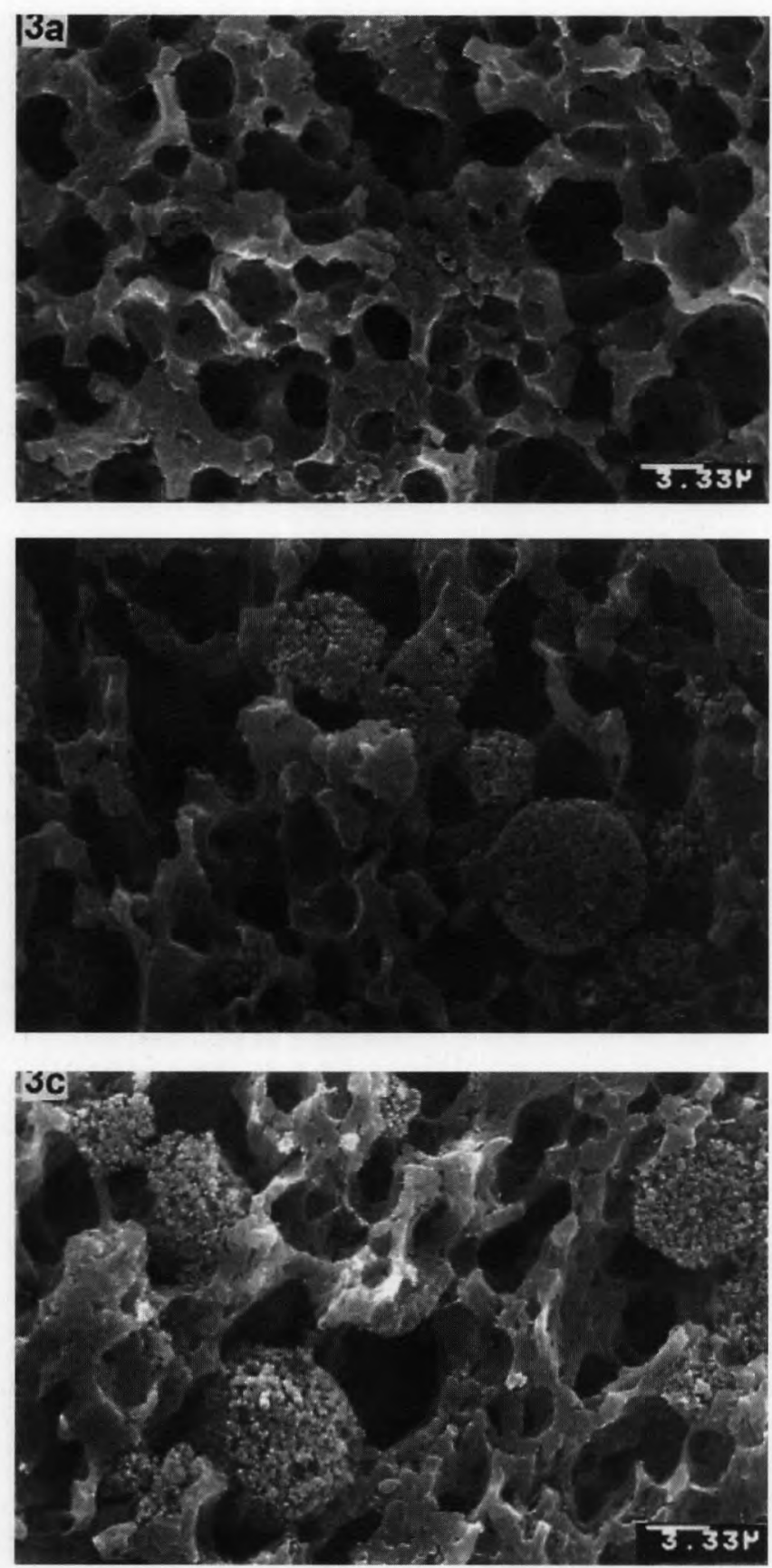

Figure 3. Microstructure des fromages additionnés des ingrédients protéiques microparticulés par SEM (3000 X) (a) fromage témoin, (b) fromage CPL 6,7 et (c) fromage CPL 4,2.

Figure 3. Microstructure of cheese fortified with whey proteins microparticulated by SEM (3000 X) (a) cheese control (b) cheese WPC 6.7 and (c) cheese WPC 4.2. 
comparativement à un cheddar normal. Du fait que les protéines du lactosérum dénaturées ont la capacité de retenir l'eau, on peut observer de grandes quantités d'eau autour des agrégats de protéines dénaturées de taille supérieure à $10 \mu \mathrm{m}$ (figure $2 b$ ).

\section{CONCLUSION}

Cette étude démontre qu'il est possible de produire des particules sphériques à partir d'agrégats de protéines du lactosérum dénaturées thermiquement et microparticulées par microfluidisation.

L'addition des protéines du lactosérum dénaturées et microparticulées au lait de fromagerie a permis une augmentation importante des rendements fromagers. La composition et la texture des fromages produits à partir de lait additionné de CPL 4,2 et CPL 6,7 ont été modifiées. Les fromages étaient en général plus humides et une partie importante des protéines microparticulées a été retenue dans la matrice fromagère. Une diminution de la rétention des lipides dans les fromages causée par la présence des CPL a été observée. L'examen par microscopie électronique des fromages a permis de visualiser la structure sphérique et globulaire des agrégats de protéines du lactosérum. Les fromages expérimentaux présentaient un réseau caséique plus ouvert et moins uniforme qu'un cheddar normal.

Les résultats obtenus nous permettent de croire que cette technologie pourrait être optimisée dans le cas des fromages allégés. En effet, les pertes en lipides et protéines sont associées à un faible rapport protéines/lipides ( $\mathrm{si}$ on considère les ingrédients protéiques microparticulés comme la matière grasse), il serait donc intéressant de voir quel genre de produit allégé on obtient en standardisant notre rapport protéines/lipides à 0,70 par l'ajout de caséines (poudre de lait écrémé). On aurait un fromage avec un bon rapport protéines/lipides, mais avec un contenu en lipides réduit.

\section{REMERCIEMENTS}

Nous remercions, le Dr M Kalab du centre de recherche sur les aliments, Agriculture Canada, Ottawa, d'avoir effectué les analyses par microscopie électronique.

Cette étude a été financée par le programme Synergie, ministère de l'Industrie du Commerce, de la Science et de la Technologie (MICST).

\section{RÉFÉRENCES}

[1] Abrahansen R.K., Cheese making from milk fortified with ultrafiltrated whey protein concentrate, Milchwissenschaft 34 (1979) $1-4$.

[2] Anonyme (1992) Natural and Process Cheese, Nutra Sweet Company, Chicago, Illinois, États-Unis.

[3] AOAC (1990) Official methods of analysis of the association of official analytical chemists, 15 th ed, Arlington, Virginie, États-Unis.

[4] Baldwin K.A., Baer R.J., Parsons J.G., Seas S.W., Spurgeon K.R., Torrey G.S., Evaluation of yield and quality of cheddar cheese manufactured from milk with added whey protein concentrate, J. Dairy Sci. 69 (1986) 2543-2550.

[5] Banks J.M., Muir D.D., Effect of incorporation of denatured whey protein on the yield and quality of cheddar cheese, J. Soc. Dairy Technol. 38 (1985) 27-32.

[6] Barbano D.M., Sherbon J.W., Cheddar cheese yield in New York, J. Dairy Sci. 67 (1984) 1873-1883.

[7] Bernal V., Jelen P., Thermal stability of whey proteins. A calorimetric study, J. Dairy Sci. 68 (1985) 2847-2852.

[8] Brown J.R., Emston C.A., Incorporation of ultrafiltration concentrated whey solids into Cheddar cheese for increase yield, J. Dairy Sci. 65 (1982) 2391-2395.

[9] Buchheim W., Jelen P., Microstructure of heat-coagulated whey protein curd, Milchwissenschaft 31 (1976) 589-592.

[10] Callanan T., Recovery of milk constituents in cheesemaking, IDF Special Issue Factors affecting the yield of cheese, International Dairy Federation, Bruxelles, Belgique, 9301, 1993, 48-52. 
[11] Cheftel J.C., Lorient D., Les propriétés fonctionnelles des protéines laitières et leurs améliorations, Lait 62 (1982) 435-483.

[12] de Wit J.N., Structure and functional behaviour of whey proteins, Neth. Milk Dairy J. 35 (1981) 47-64.

[13] Emmons D.B., Economic importance of cheese yield. IDF Special Issue. Factors affecting the yield of cheese, International Dairy Federation, Bruxelles, Belgique 9301, 1993a, 10-11.

[14] Emmons D.B., Definition and expression of cheese yield. IDF Special Issue. Factors affecting the yield of cheese, International Dairy Federation, Bruxelles, Belgique, 9301, 1993b, 12-20.

[15] Emmons D.B., Ernstrom C.A., Lacroix C., Verret P., Yield formulae. Special Issue. Factors affecting the yield of cheese, International Dairy Federation, Bruxelles, Belgique, 9301, 1993, 21-47.

[16] FIL, Lait, détermination de la teneur en azote partie 3 et 4 . Norme FIL internationale, Bruxelles, Belgique, 1993, 20.

[17] FIL, Poudres de protéines laitières, détermination de l'indice de solubilité de l'azote. Norme FIL internationale, Bruxelles, Belgique, 1995, 173.

[18] Jensen G.K., Stapelfeldt H., Incorporation of whey proteins in cheese. Including the use of ultrafiltration. IDF Special Issue. Factors affecting the yield of cheese, International Dairy Federation, Bruxelles, Belgique, 9301, 1993, 88-108.

[19] Langton M., Hermansson A.M., Fine-stranded and particulate gels of $\beta$-lactoglobulin and whey protein at varying $\mathrm{pH}$, Food Hydrocolloid 6 (1992) 523-539.
[20] Lawrence R.C., The use of ultrafiltration technology in cheesemaking, Bull. Int. Dairy Fed. Bruxelles, Belgique (1987) B136.

[21] Lawrence R.C., Incorporation of whey proteins in cheese. IDF Special Issue Factors affecting the yield of cheese. International Dairy Federation, Bruxelles, Belgique, 9301, 1993, 79-87.

[22] Lawrence R.C., Lelievre J., Manufacture of cheese from milk concentrated by ultrafiltration, J. Dairy Res. 55 (1988) 465-478.

[23] Lelievre J., Incorporation of size reduced dispersions of whey proteins into Mozzarella cheese. Proceeding 23rd IDF congress, Montréal, 1990, p. 517.

[24] MAPAQ, Contrôle de la qualité des produits laitiers. Gouvernement du Québec, Bibliothèque nationale du Québec, 1984, p. C 4-41.

[25] Paquin P., Lebeuf Y., Richard J.P., Kalab M., Microparticulation of milk proteins by high pressure homogeneization to produce a fat substitute. Protein and fat globule modifications. IDF special issue. International Dairy Federation, Bruxelles, Belgique, 9303, 1993, 389-396.

[26] Robin O., Paquin P., Evaluation of the particle size of fat globules in a milk model emulsion by photon correlation spectroscopy, J. Dairy Sci. 74 (1991) 2440-2447.

[27] Singer N.S., Yaptamotx S., Latella J., Protein product base. US Patent $n^{\circ} 4,1988,734$ 287.

[28] Xiong Y.L., Dawson K.A., Wan L., Thermal agregation of $\beta$-lactoglobulin: Effect of $\mathrm{pH}$, ionic environment and thiol reagent, J. Dairy Sci. 76 (1993) 70-77. 